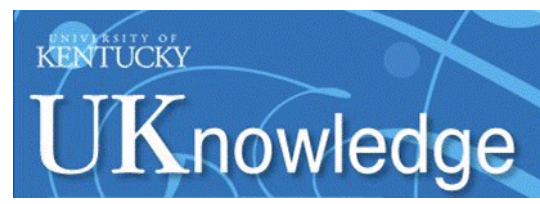

University of Kentucky

UKnowledge

$1-1999$

\title{
Numerical Simulations of Fe II Emission Spectra
}

\author{
E. M. Verner \\ University of Kentucky \\ D. A. Verner \\ University of Kentucky \\ K. T. Korista \\ University of Kentucky \\ Jason W. Ferguson \\ University of Kentucky \\ F. Hamann \\ University of California - San Diego
}

See next page for additional authors

Follow this and additional works at: https://uknowledge.uky.edu/physastron_facpub

Part of the Astrophysics and Astronomy Commons, and the Physics Commons

Right click to open a feedback form in a new tab to let us know how this document benefits you.

\section{Repository Citation}

Verner, E. M.; Verner, D. A.; Korista, K. T.; Ferguson, Jason W.; Hamann, F.; and Ferland, Gary J., "Numerical Simulations of Fe II Emission Spectra" (1999). Physics and Astronomy Faculty Publications. 144.

https://uknowledge.uky.edu/physastron_facpub/144

This Article is brought to you for free and open access by the Physics and Astronomy at UKnowledge. It has been accepted for inclusion in Physics and Astronomy Faculty Publications by an authorized administrator of UKnowledge. For more information, please contact UKnowledge@lsv.uky.edu. 


\section{Numerical Simulations of Fe II Emission Spectra}

Digital Object Identifier (DOI)

https://doi.org/10.1086/313171

Notes/Citation Information

Published in The Astrophysical Journal Supplement Series, v. 120, no. 1, p. 101-112.

(C)1999. The American Astronomical Society. All rights reserved.

The copyright holder has granted permission for posting the article here.

Authors

E. M. Verner, D. A. Verner, K. T. Korista, Jason W. Ferguson, F. Hamann, and Gary J. Ferland 
The Astrophysical Journal Supplement Series, 120:101-112, 1999 January

(C)1999. The American Astronomical Society. All rights reserved. Printed in U. S. A.

\title{
NUMERICAL SIMULATIONS OF Fe II EMISSION SPECTRA
}

\author{
E. M. Verner,${ }^{1}$ D. A. Verner, ${ }^{1}$ K. T. Korista, ${ }^{1,2}$ J. W. Ferguson,,${ }^{1,3}$ F. Hamann, ${ }^{4}$ and G. J. Ferland ${ }^{1}$ \\ Received 1998 April 23; accepted 1998 August 10
}

\begin{abstract}
This paper describes the techniques that we have used to incorporate a large-scale model of the $\mathrm{Fe}^{+}$ ion and resulting Fe II emission into CLOUDY, a spectral synthesis code designed to simulate conditions within a plasma and model the resulting spectrum. We describe the numerical methods we use to determine the level populations, mutual line overlap fluorescence, collisional effects, and the heatingcooling effects of the atom on its environment. As currently implemented, the atom includes the lowest 371 levels (up to $11.6 \mathrm{eV}$ ) and predicts intensities of 68,635 lines. We describe our data sources, which include the most recent transition probabilities and collision strengths. Although we use detailed fits to temperature-dependent collision strengths where possible, in many cases the uncertain $\bar{g}$ approximation is the only source for collision data. The atom is designed to be readily expanded to include more levels and to incorporate more accurate sets of collision and radiative data as computers grow faster and the atomic databases expand. We present several test cases showing that the atom goes to LTE in the limits of high particle and radiation densities. We give an overview of general features of the Fe II spectra as their dependencies on the basic parameters of our models (density, flux, microturbulent velocity, the $\mathrm{Fe}$ abundance, and Ly $\alpha$ pumping). Finally, we discuss several applications to active galactic nuclei to illustrate the diagnostic power of the Fe II spectrum and make some predictions for UV observations.
\end{abstract}

Subject headings: atomic processes - ISM: atoms - line: formation - methods: numerical

\section{INTRODUCTION}

The Fe II emission spectrum is a ubiquitous one, appearing in such objects as stars, protostellar disks, $\mathrm{H}$ II regions, novae, supernova remnants, active galaxies, and quasars. The high abundance of iron in the universe and the very rich spectrum of Fe II explain why this is so. Many tens of terms, corresponding to hundreds of levels, contribute. Laboratory spectroscopy is not complete, and only in the past few years have many of the radiative and collision rates been determined. First optical and then UV and IR spectral windows have become available to measure the full spectrum in astrophysical sources. The atom is far from equilibrium in most emission-line objects, so the spectrum is sensitive to the detailed local conditions. A complete simulation of the physical processes affecting the $\mathrm{Fe}$ II spectrum would make it possible to deduce the density, temperature, and iron abundance of the emitting regions. This is the major long-term goal of our program.

This goal is difficult because the physics governing Fe II emission is complex. A variety of processes affect the spectrum, including collisional excitation, pumping by the incident continuum, fluorescence by line overlap, and line transport. It is this sensitivity to details that makes Fe II a potentially powerful probe of physical conditions in the emitting gas. Even the results are not simple, however; tens of thousands of lines often form a pseudocontinuum that can only be compared with observations after a complete spectral synthesis. Fe II emission presents a challenge at the very forefront of computational nebular astrophysics.

\footnotetext{
${ }^{1}$ University of Kentucky, Department of Physics and Astronomy, Lexington, KY 40506-0055.

${ }^{2}$ Western Michigan University, Department of Physics, Kalamazoo, MI 49008.

${ }^{3}$ Wichita State University, Department of Physics, Wichita, KS 672600032.

${ }^{4}$ University of California, San Diego, Center for Astrophysics and Space Science, La Jolla, CA 92093-0111.
}

There have been a number of pioneering theoretical investigations into $\mathrm{Fe}$ II emission spectra in active galactic nuclei (AGNs). For example, Phillips (1978a, 1978b) discussed continuum pumping as one of the excitation mechanisms that is responsible for the Fe II emission. Brown, Jordan, \& Wilson (1979) and Penston et al. (1983) suggested that Ly $\alpha$ pumping might excite high-excitation transitions in late-type and symbiotic stars. Penston (1987) showed that this process can be very important in the overall $\mathrm{Fe}$ II flux from the symbiotics and might, therefore, contribute substantially to the strong Fe II emission from AGNs. Netzer \& Wills (1983) and Wills, Netzer, \& Wills (1985) developed the most detailed models of the Fe II emission from the broad emission line region of quasars. These models took into account the processes of continuum and line pumping, including Ly $\alpha$ pumping and $\mathrm{Fe}$ II self-fluorescence. The models were based on atomic data available in the 1980s and assumed LTE population for some Fe II levels.

In contrast to the situation in AGN research, there is a large body of recent theoretical investigations of $\mathrm{Fe}$ II emission in stellar sources. Applications include supernova envelopes (Hauschildt et al. 1996; Li \& McCray 1996; Kozma \& Fransson 1998a, 1998b; Hoeflich, Wheeler, \& Thielemann 1998) and more normal stars (Lanz, Hubeny, \& Heap 1997; Young, Landi, \& Thomas 1998). This work is largely based on stellar atmosphere techniques (see, for example, Mihalas 1978), in particular using fairly exact radiative transfer methods. The approach we take here comes from a plasma and nebular physics background (see, for example, Netzer 1990) and tries very hard to do the best possible simulation of the microphysics, at the expense of a more approximate radiative transfer treatment, based on escape probability methods. This approach has advantages in both speed and portability (Kalkofen 1984). It is applicable to a wide range of astrophysical environments from $\mathrm{H}$ II regions and circumstellar envelopes to the various emission- and absorption-line regions of AGNs. We also note that for many of these applications we have little or no 


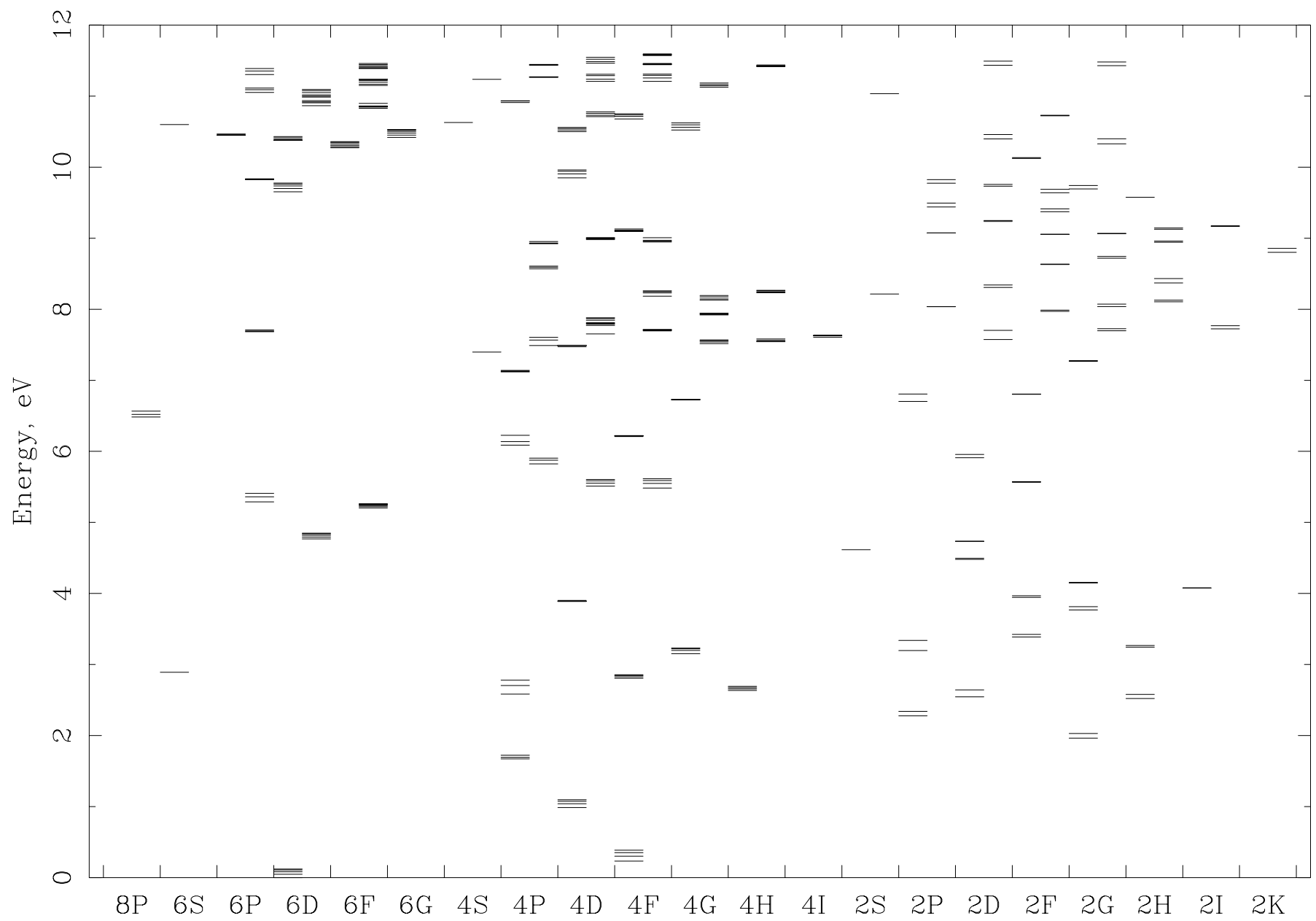

FIG. 1.-Energy distribution of the 371 lowest levels of $\mathrm{Fe}$ II that are included in our calculations. The horizontal axis shows terms ranged from octets to doublets. The vertical scale indicates energy of levels in eV. For each multiplicity and orbital momentum, even terms are on the left, and odd terms are on the right.

knowledge of the local geometry and velocity field. Therefore, the escape probabilities and Gaussian microturbulent velocities we will use are appropriate (maybe even preferable) for exploring the gross sensitivities of the line spectra to various parameters.

Here we describe the methods and approximations that we have used to incorporate a large $\mathrm{Fe}^{+}$ion and predict the resulting emission into CLOUDY, a spectral synthesis code designed to simulate conditions within a plasma and model the resulting spectrum (Ferland $\left.1996^{5}\right)$. This is a logical first step in expanding our detailed treatment of large atoms, since $\mathrm{Fe}^{+}$is the dominant stage of ionization of iron for most AGN cloud conditions and its spectrum is strong. Ferland et al. (1998) described the last publicly available version of CLOUDY, version 90 . We test and verify our models under various physical conditions, overview general features of the Fe II spectra, and discuss their applications.

\section{THE Fe II MODEL ATOM AND ATOMIC DATA}

\subsection{The Model Atom and Its Atomic Data}

Our current model of the Fe II atom includes all 371 levels with energies below $93,487.650 \mathrm{~cm}^{-1}$, or $11.59 \mathrm{eV}$. All

\footnotetext{
${ }^{5}$ CLOUDY is available at http://www.pa.uky.edu/ gary/cloudy.
}

level energies are experimental (Johansson 1978; S. Johansson 1995, private communication) and should be quite accurate. Figure 1 shows the energy distribution of the 371 lowest levels of $\mathrm{Fe}^{+}$. The horizontal axis shows terms ranged from octets to doublets. The vertical scale indicates energy of levels in electron volts. For each multiplicity and orbital momentum, even terms are on the left and odd terms are on the right.

Figure 2 shows how the number of lines predicted by our model depends on the wavelength. All predicted lines are shown in the upper curve, and the lower curve shows the permitted lines. The fact that the densities of lines are different for shorter and longer wavelengths shows that a different approach must be taken for IR and UV lines. For IR, individual lines can be identified. For visible wavelengths, many lines are blended. For the UV, a complete spectral synthesis must be used because the density of lines is large enough for the lines to form a blended pseudocontinuum. The density of lines can reach several hundreds per angstrom in the UV. This synthesis approach will be used in $\S 4$.

Transition probabilities are taken from theoretical calculations by Nahar (1995, allowed transitions) and Quinet, Le Dourneuf, \& Zeippen (1996, forbidden transitions) and supplemented by data from compilations by Fuhr, Martin, \& 


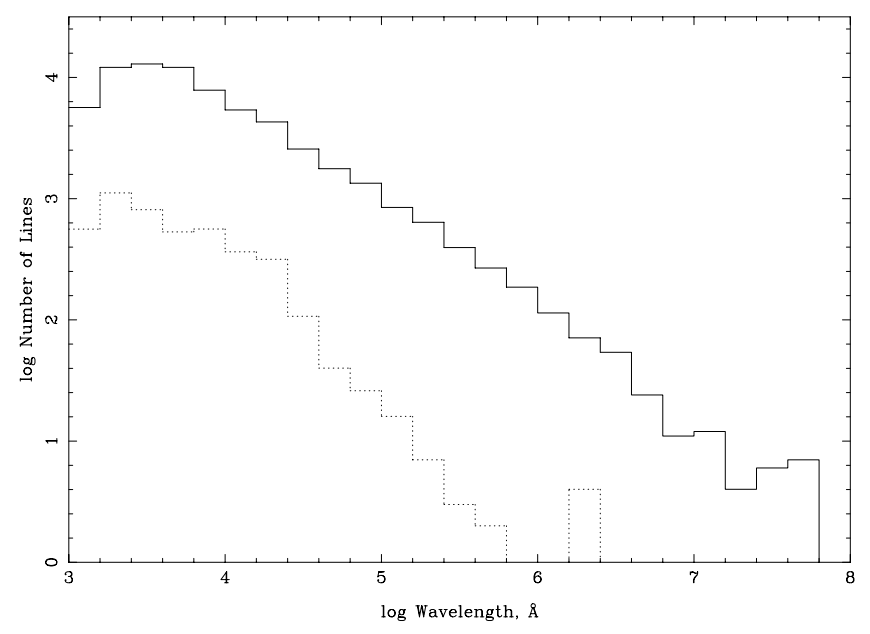

FIG. 2.- - Log-log distribution of Fe II lines on wavelength (solid line, all lines; dashed line, permitted lines).

Wiese (1988) and Giridhar \& Arellano Ferro (1995). Transition probabilities for all intercombination lines not covered by these compilations are taken from the Kurucz (1995) database. Uncertainties are generally smaller than $20 \%$ for strong permitted lines but can be larger than $50 \%$ for weak permitted and intercombination lines. For the forbidden transitions of interest, uncertainties are expected to be less than $50 \%$.

Collision strengths for all quartet and sextet transitions among the 142 levels (10,011 lines) are taken from Zhang \& Pradhan (1995). For other lines, we use interpolations and $\bar{g}$ approximation (Mewe 1972). It is hard to judge the uncertainties in the theoretical calculations, but they might be within $30 \%$ for strong lines and 0.5 dex for weak and $\bar{g}$ lines.

\subsection{Balance Equations}

The first step in modeling the Fe II emission spectrum is to calculate the atom's level populations. A realistic model requires a sufficiently large number of levels and must include all excitation and de-excitation mechanisms. We assume that the atom is in steady state and solve for all level populations by balancing processes that populate and depopulate the levels.

Let $n_{i}$ be the number density (in $\mathrm{cm}^{-3}$ ) of $\mathrm{Fe}^{+}$ions in level $i$. For steady state conditions, $n_{i}$ is constant with time, and the number of transitions into level $i$ is equal to the corresponding number of transitions out per second per cubic centimeter. For the $i$ th level population, the complete form of the rate equation is

$$
\begin{aligned}
\frac{d n_{i}}{d t}= & \sum_{u=i+1}^{371}\left[\left(A_{u i}^{\prime}+q_{u i} n_{e}\right) n_{u}-\left(B_{i u}^{\prime}+q_{i u} n_{e}\right) n_{i}\right] \\
& +\sum_{l=1}^{i-1}\left[\left(B_{l i}^{\prime}+q_{l i} n_{e}\right) n_{e}-\left(A_{i l}^{\prime}+q_{i l} n_{e}\right) n_{i}\right],
\end{aligned}
$$

where $A_{u i}^{\prime}$ is the effective spontaneous transition probability $A_{u i}^{\prime}=A_{u i}\left(\varepsilon_{u i}+\delta_{u i}\right), A_{u i}$ is the Einstein coefficient, $\varepsilon_{u i}$ is the escape probability, and $\delta_{u i}$ is the destruction probability (Ferland et al. 1992).

The induced rate of transitions upward from $i$ is $B_{i u}^{\prime}$ and is given by $B_{i u} \bar{J}_{i u}$, where $B_{i u}$ is the induced transition probability and $\bar{J}_{i u}$ is the mean intensity averaged over the line profile. $\bar{J}_{i u}$ includes the attenuated incident continuum and net radiation field produced by other lines. The flux $\bar{J}_{i u}$ is calculated self-consistently, again using the escape probability formalism (see Elitzur \& Netzer 1985).

In equation (1), $q_{i u}$ is the collisional excitation rate coefficient (in $\mathrm{cm}^{3} \mathrm{~s}^{-1}$ ), and $n_{e}$ is the electron density. We use our fits to the temperature dependence of the electron collision strengths to obtain $q$. Collisional de-excitation rates are obtained by detailed balance, $q_{i u}=q_{u i} \exp (h v / k T) g_{i} / g_{u}$, where the $g$ 's are the statistical weights. Proton and hydrogen atom collisions are neglected but will not be important if carbon is at least once ionized, as should be true in any region in which $\mathrm{Fe}$ is ionized to $\mathrm{Fe}^{+}$.

The level populations can be obtained numerically by solving the set of algebraic equations by matrix inversion. The system of equations given by equation (1) is overdetermined. We replace the balance equation for the ground level with the conservation equation $\sum_{i} n_{i}=n\left(\mathrm{Fe}^{+}\right)$, where $n\left(\mathrm{Fe}^{+}\right)$is the total number density of $\mathrm{Fe}^{+}$ions, and find the level populations by solving the matrix. However, the matrix coefficients in turn indirectly depend on the $\mathrm{Fe}^{+}$ level populations. Thus, the final solution of the problem is obtained by iteration, with the feedback effects of the atom on the environment explicitly taken into account.

\subsection{Line Transfer}

All lines are transported using the escape probability formalism (Elitzur 1984, 1992) in the current version of our model. The form of the coding was done to allow eventual incorporation of exact transfer methods when computers become fast enough.

Both continuum and line pumping are considered in the radiative rates. This includes pumping by all lines of other atoms and ions included in the simulation as well as selfpumping by overlapping Fe II lines. Wills, Netzer, \& Wills (1985) stressed the importance of the last effect. In the escape probability formalism, radiation produced by a line is not counted as an excitation process for that particular line but is one for all other lines. Line pumping of a particular transition is efficient if the wavelength of the Fe II line coincides with another optically thick line. In the master set of equations, the loss of a photon in a particular transition due to absorption in another transition counts in the destruction probability of the first and fluorescence of the second.

Selective excitation of high-lying levels by Ly $\alpha$ pumping might be very important in stars and AGNs (Penston et al. 1983). We treat the $L y \alpha$ line as a trapezoid with the profile and line width given by Adams (1972):

$$
\Delta v=\Delta v_{\text {Dop }} \times 2.3(a \tau)^{1 / 3},
$$

where $\Delta v_{\text {Dop }}$ is the Doppler width, $a$ is the damping parameter, and $\tau$ is the line-center optical depth. The excitation temperature of the $\mathrm{Ly} \alpha$ line is determined by the separate solution of the hydrogen level populations (Ferguson \& Ferland 1997), with Fe II included as a Ly $\alpha$ loss mechanism to ensure energy conservation. The number of $\mathrm{Fe}$ II lines that can be pumped by $\operatorname{Ly} \alpha$ is very sensitive to the $\operatorname{Ly} \alpha$ optical depth and resulting line width, to the excitation temperature of $\operatorname{Ly} \alpha$, and to the electron temperature, since this determines the lower level populations of $\mathrm{Fe}^{+}$. The number of lines can range from only a few lines to tens of lines. 
For all other optically thick lines, we use a simplified rectangular profile to calculate the line widths. Again, the line widths are a function of the optical depths, which are only known after several iterations. We use the line profile suggested by Elitzur \& Ferland (1986):

$$
\Delta v= \begin{cases}\Delta v_{\text {Dop }} \times(2 \pi)^{1 / 2}, & \tau \leq 4.81, \\ \Delta v_{\text {Dop }} \times 2(\ln \tau)^{1 / 2}, & 4.81<\tau \leq 10^{4}, \\ \Delta v_{\text {Dop }} \times 2.3(a \tau)^{1 / 3}, & \tau>10^{4} .\end{cases}
$$

\subsection{Heating-cooling}

The balance of all cooling and heating processes determines the electron temperature $T_{e}$. We define heating and cooling relative to the continuum (Osterbrock 1989), so only processes that change the electron kinetic energy count. The total collisional cooling (in ergs $\mathrm{cm}^{-3} \mathrm{~s}^{-1}$ ) produced by $\mathrm{Fe}^{+}$is

$$
\Lambda_{\mathrm{Fe} \mathrm{II}}=\sum_{l=1}^{N-1} \sum_{u=l+1}^{N} h v_{u l} n_{e}\left(n_{l} q_{l u}-n_{u} q_{u l}\right),
$$

where $h v_{u l}$ is the energy of the transition. If a level inversion occurs, so that the population of a level relative to its LTE value increases with excitation potential, the cooling will be negative and the atom will act to heat the gas. This will occur in some of the following models. This happens since some pumping mechanisms selectively populate very highly excited levels, which are then collisionally de-excited, effectively converting radiative into kinetic energy.

Fe II often dominates the thermal equilibrium of a cloud and can account for well over $90 \%$ of the cooling in some conditions. In such cases, the temperature predictorcorrector must have a very good analytic estimate of the derivative of the net $\mathrm{Fe}$ II cooling to apply the next temperature correction. We have tried various prescriptions for this derivative for large multilevel atoms. We found that taking the excitation temperature as the energy between the upper level and the ground level can best represent the derivative of the cooling between two levels. Then the cooling derivative at constant ionization will be

$$
\left.\frac{d \Lambda}{d T}\right|_{\text {ion }}=\sum_{l=1}^{N-1} \sum_{u=l+1}^{N} h v_{u, l} n_{e}\left(n_{l} q_{l, u}-n_{u} q_{u, l}\right)\left(\frac{h v_{u, 1}}{T_{e}^{2}}-\frac{1}{2 T_{e}}\right) .
$$

In practice, the ionization often changes with temperature and it is necessary to further iterate on the solution. The calculations are repeated until a stable solution is obtained. Typically 10 iterations were required. Specific examples of the effects of $\mathrm{Fe}$ II on the thermal structure of a cloud are given below.

\subsection{Fe II and Its Interaction with the Environment}

The Fe II model has been incorporated into the development version of the radiative-collisional code CLOUDY (Ferland 1996; Ferland et al. 1998). Ferland and coworkers give an overview of the methods and approximations used by the code. We briefly summarize parts relevant to Fe II here.

An optically thick slab is divided into a large number of mesh points, referred to as zones, chosen to be thin enough for the ionization and temperature to be nearly constant across each bin. We then solve the set of statistical equi- librium equations (see eq. [1]) in each zone. Simultaneously, the electron temperature $T_{e}$, the local field $\bar{J}_{v}$, the electron density $n_{e}$, and the ionization balance of all elements are all calculated self-consistently. These result in a net heating or cooling of the gas, with all $\mathrm{Fe}$ II interactions included. The electron temperature is determined by making the difference between the heating and cooling rates smaller than a preset tolerance. The local radiation field is determined from the level populations of all species and the local opacity. We recalculate all of these values and iterate to convergence. The final spectrum is the integration over the computed structure.

There are fundamental uncertainties in the physics of $\mathrm{Fe}$ II emission due to errors in the basic atomic data. These are both systematic and random errors and are hard to evaluate. However, our calculation has energy balance as its foundation, and this limits the largest possible excursion due to errors in the basic rates. The purposes of the large numerical simulations are basically to determine how much energy is absorbed from the incident continuum and then to find in which channels the energy will be reradiated. Past experience shows that substantial uncertainties in basic rates seldom carry over linearly into the computed spectrum.

\section{THE Fe II ATOM'S APPROACH TO LTE}

Before attempting to simulate the $\mathrm{Fe}$ II spectrum in a nonequilibrium environment, we verified that the model atom goes to local thermodynamic equilibrium (LTE) in four limiting cases. We define the departure coefficients as a ratio $b_{i}=n_{i} / n_{i, \mathrm{LTE}}$, where $n_{i}$ is the actual population of $i$ th level and $n_{i, \mathrm{LTE}}$ is the population of the $i$ th level as specified from the Boltzmann distribution. Each of these limiting cases has (different) parameters chosen so that $\mathrm{Fe}$ would have a significant fraction singly ionized.

\subsection{Collision-dominated Case}

Figure 3 shows a test case in which collisional processes are dominant. The iron abundance has been set to a very large number, $(\mathrm{Fe} / \mathrm{H})=100$, to ensure that the $\mathrm{Fe}^{+}$dominates the calculation. All of the radiative processes are actually included, but the external radiation flux was set to

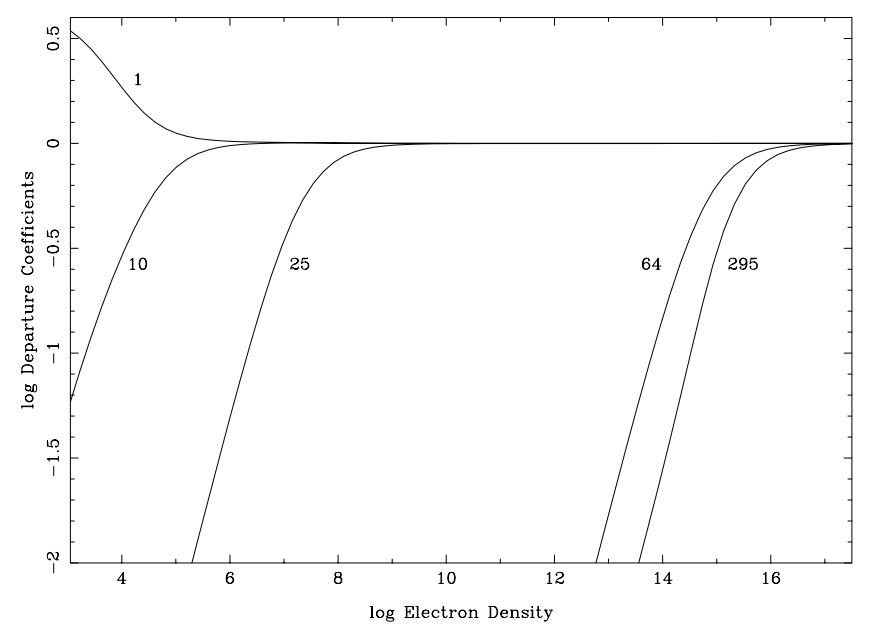

FIG. 3.-Departure coefficients of selected Fe II levels vs. electron density. Population of all levels reaches LTE at densities above $10^{16} \mathrm{~cm}^{-3}$. 
a very low intensity. The model is of a very thin cell of gas that is optically thin in the lines and continuum, i.e., the column density is kept small enough for optical depth effects to be unimportant. The level populations are determined by solving the full set of equations of statistical equilibrium for a preset electronic temperature $T_{e}=5000 \mathrm{~K}$. Departure coefficients for the ground state (first level) and for the 10th, 25th, 64th, and 295th levels are shown as a function of the electron density $n_{e}$. Note that the first 63 levels have even parity, and therefore radiative transitions between these levels have very low probability. As a result, these levels come into LTE at much lower densities than the upper levels starting from the 64th, which can decay by permitted transitions.

For low densities, the ground state is overpopulated relative to its LTE value. This is because upward collisional processes are much slower than downward radiative processes. When the densities are high, these rates become equal and the departure coefficients approach unity. Similarly, highly excited levels are underpopulated relative to their LTE values at low densities, since radiative decays to lower levels are faster than collisional processes. For densities $n_{e}>10^{16} \mathrm{~cm}^{-3}$, collisional processes completely dominate the rates, and the entire atom reaches LTE.

\subsection{Radiation-dominated Case}

Figure 4 shows a series of tests in which the external radiation field becomes increasingly dominant. This culminates in the atom going to equilibrium in the case of a true blackbody radiation field. Again, the full set of equations coupling the levels are solved. The density is low enough $\left(n_{\mathrm{H}}=10^{9} \mathrm{~cm}^{-3}\right)$ and the radiation field becomes intense enough for spontaneous and induced radiative processes to dominate the atom's level populations. The Fe abundance is set to the same high value as in the collisional model described above. The electron temperature is a constant $8000 \mathrm{~K}$. Again the model is of a very thin cell of gas, so that all lines and continuum are optically thin. The gas is exposed to a blackbody continuum with a color temperature of $T_{c}=8000 \mathrm{~K}$ but with various values of the energy density of the radiation field. The equivalent energy density temperature is $T_{u}=(u / a)^{1 / 4}$, where $u$ is the total

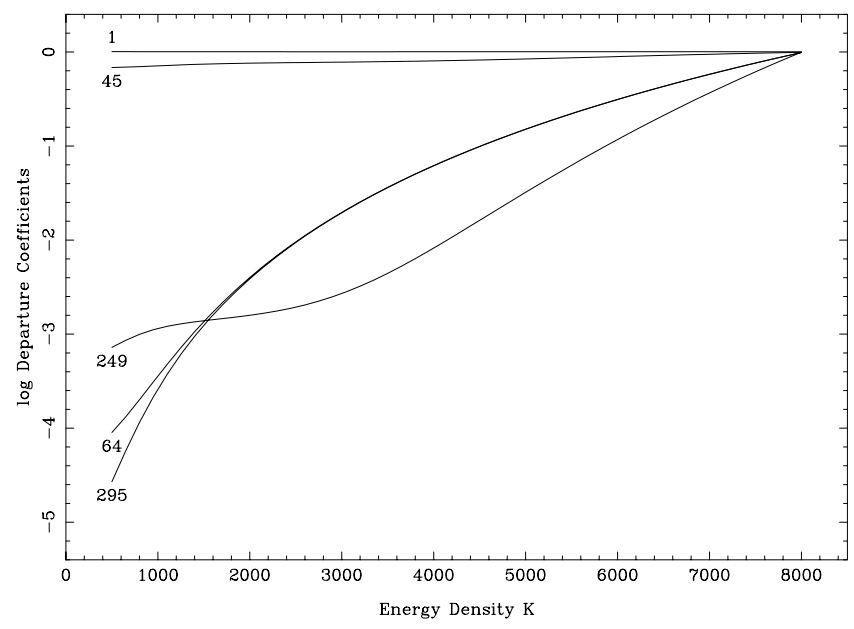

FIG. 4.-Departure coefficients of selected Fe II levels vs. energy density of radiation field. Population of all levels reaches LTE with increasing energy density. energy density in all radiation (in ergs $\mathrm{cm}^{-3}$ ) and $a$ is the Stefan radiation density constant.

For this electron density, the lowest 63 even levels are close to LTE for any external radiation field, but the higher levels, from which permitted transitions exist, are far from LTE. For low-energy densities, the level populations of excited states are below their LTE value, while the ground level is overpopulated. Increasing the radiation field has the effect of increasing populations of high levels as pumping of these levels from ground grows more important. A radiation field given by Planck's law (i.e., $T_{u} \equiv T_{c}$ ) forces the ionization and level populations of atom to LTE in much the same way that high electron densities do. In the limit where $T_{u} \equiv T_{c}$, the departure coefficients reach unity and the atom goes to the strict thermodynamic equilibrium limit.

\subsection{Large Optical Depths}

The third test checks that the Fe II atom goes to LTE with increasing continuum optical depth at $912 \AA$ (Fig. 5). The model assumes a constant temperature of $15,000 \mathrm{~K}$, a very high iron abundance, $(\mathrm{Fe} / \mathrm{H})=10^{4}$, a gas density $n_{\mathrm{H}}=$ $10^{9} \mathrm{~cm}^{-3}$, and a very low ionization radiation density. Figure 5 shows the departure coefficients for the ground state (first level) and for the 124th, 206th, and 347th levels as a function of increasing optical depth in the Lyman continuum. All of the levels reach their LTE populations at sufficiently large optical depth, where line trapping is extreme and the atom becomes effectively collisionally dominated.

\subsection{Thermal Equilibrium}

The ultimate test is that a fully iron-dominated environment equilibrates at the correct temperature and population when the radiation field approaches strict thermodynamic equilibrium (i.e., $T_{u} \equiv T_{c}$ ). Particles and light must come into equilibrium in this limit, and this simultaneously tests the ionization, excitation, and thermal effects of the model atom. These tests are similar to the applications to quasars below in that the electron temperature is determined self-consistently from the balance between heating and cooling. The conditions are similar to those in $\S 3.2$ with the exception that the electron tem-

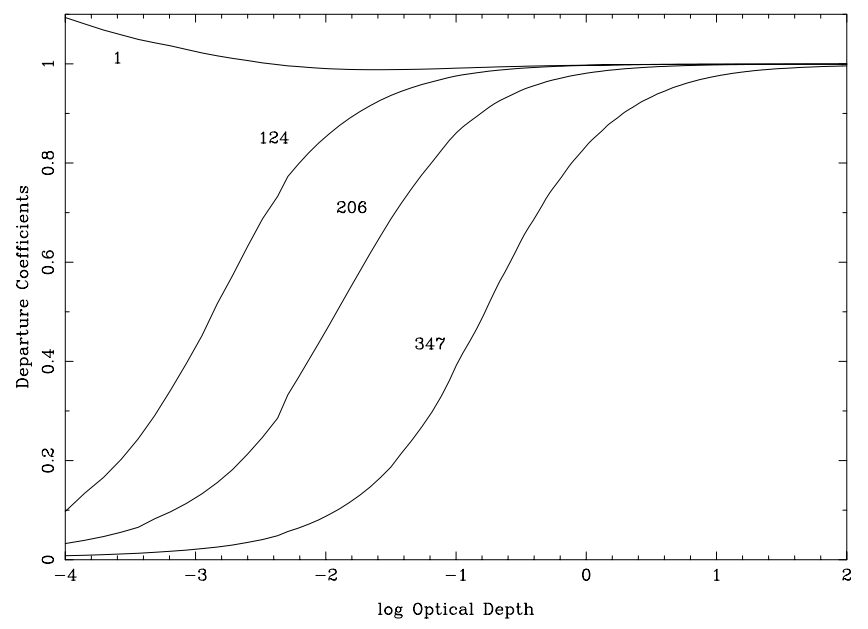

FIG. 5.-Departure coefficients of selected Fe II levels vs. the $912 \AA$ continuum optical depth of the cloud. Population of all levels reaches LTE at optical depth higher than $10^{4}$. 


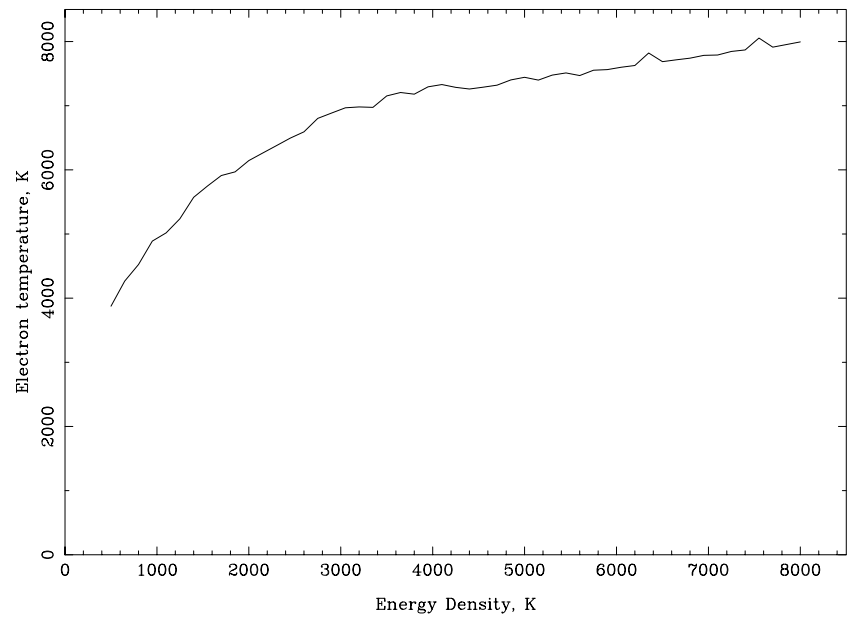

Fig. 6.-Calculated electron temperature vs. the energy density temperature. They reach the same value when the energy density temperature reaches the $8000 \mathrm{~K}$ blackbody temperature.

perature is determined self-consistently. Figure 6 shows how the calculated electron temperature changes with the energy density temperature and reaches the same value as the energy density temperature at $8000 \mathrm{~K}$. In this limit, we have recovered the limit where the photons and particles have come into complete statistical equilibrium, verifying that the calculation is fully self-consistent.

\section{APPLICATIONS}

Observationally, the $\mathrm{Fe}$ II spectrum is rich across a broad wavelength range from the UV to IR, and various astronomical objects show quite different types of $\mathrm{Fe}$ II emission spectra. The goal is to determine the physical conditions in $\mathrm{Fe}$ II line-forming regions by modeling the $\mathrm{Fe}$ II emission. In this section, we discuss general features of the Fe II spectra as they depend on the basic parameters of our models. We will primarily be concerned here with broad emission line regions (BELRs) of AGNs. We intentionally consider many different models to illustrate the greatest diversity of possible Fe II emission.

\subsection{Overview of the Cloud Structure}

This section is gives an overview of the effects of Fe II emission upon the thermal structure of a typical BELR cloud. The conditions were chosen so that low ionization lines like $\mathrm{Fe}$ II will dominate the emission. The clouds were illuminated by a spectral energy distribution thought typical of AGNs (Mathews \& Ferland 1987). Solar abundances and constant density are used. The hydrogen density is $n_{\mathrm{H}}=10^{12} \mathrm{~cm}^{-3}$, the column density is $10^{23} \mathrm{~cm}^{-2}$, the flux of hydrogen ionizing photons at the illuminated face is $3 \times 10^{19} \mathrm{~cm}^{-2} \mathrm{~s}^{-1}$, and these correspond to an ionization parameter $U(\mathrm{H})=\Phi_{\mathrm{H}} / n_{\mathrm{H}} c$ of $10^{-3}$. Figures 7-9 show how the electron temperature and the ionization fractions of $\mathrm{H}^{0}$, $\mathrm{H}^{+}, \mathrm{He}^{0}, \mathrm{He}^{+}, \mathrm{He}^{+2}$, and $\mathrm{Fe}^{0}-\mathrm{Fe}^{+4}$ change with depth into cloud.

As is typical of an X-ray illuminated atmosphere, a large column density of partially ionized gas with a warm temperature exists beyond the hydrogen ionization front. This is the combined result of penetrating X-rays and photoionization of the $n=2$ level of hydrogen by the Balmer continuum. The latter depends exponentially on the electron

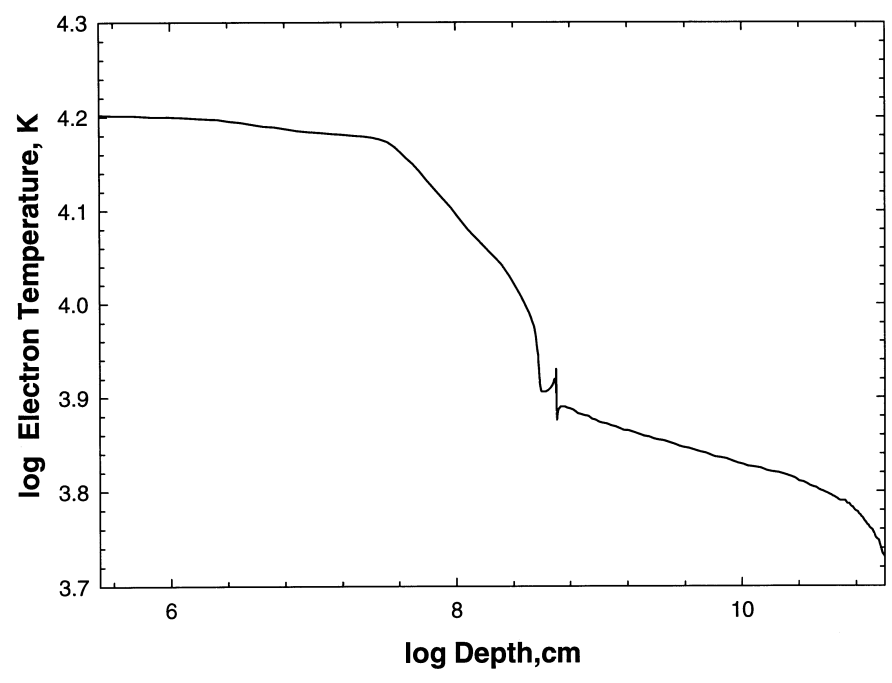

FIG. 7.- $-\log$ of the electron temperature (K) vs. depth within the cloud (cm).

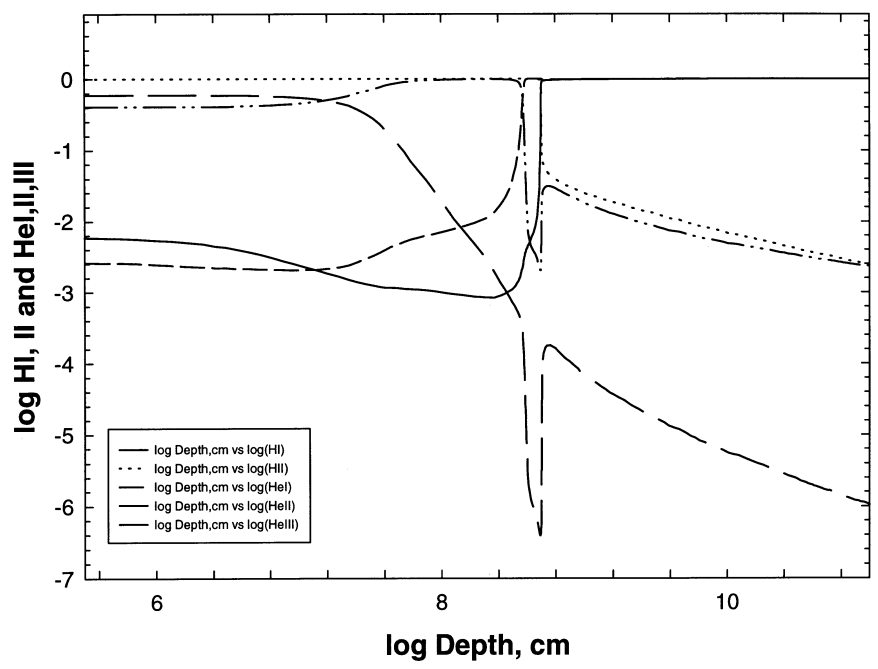

FIG. 8. - $\log$ of the ionization fractions of $\mathrm{H} \mathrm{I}, \mathrm{H} \mathrm{II}, \mathrm{He}$ I, He II, and $\mathrm{He}$ III vs. depth within the cloud (in $\mathrm{cm}$ ). The ionization fronts appear as discontinuities but are well-resolved in the numerical calculations.

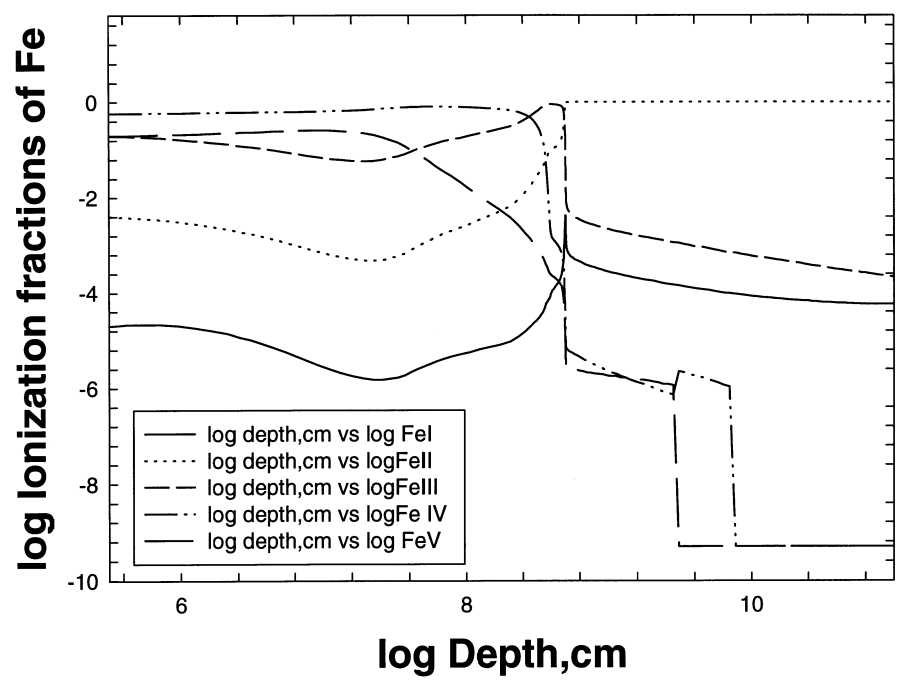

FIG. 9. - $\log$ of the ionization fractions of $\mathrm{Fe}$ I-Fe v vs. depth within the cloud (in $\mathrm{cm}$ ). Sharp ionization fronts are not present, as is typical of ionization structures of the heavy elements and described by Ferland \& Persson (1989). 
temperature, since this affects the population of the $n=2$ level. There is also strong feedback between Fe II emission and the hydrogen $n=2$ population because iron provides an extra loss mechanism through Ly $\alpha$ pumping.

The Fe II lines are strong in many quasars, and we expect that in some conditions Fe II can dominate the heating or cooling of the cloud. Fe II is an efficient coolant because of its large number of lines, offering many channels for emission despite the strong trapping due to large optical depths. In situations in which collisional de-excitation of excited levels is significant, it can heat the gas as well. These effects are fundamental, since the calculations described in this paper have energy balance as their foundation: the energy in the incident continuum is only being repartitioned among the various lines and continua that are emitted. If $\mathrm{Fe}$ II heating or cooling alters the thermal balance of the cloud, the rest of the spectrum will also change to maintain energy conservation. Thus, the details of Fe II emission will feed back into other lines emitted from the same region such as the Balmer lines or $\mathrm{Mg}$ II $\lambda 2798$.

Figures 9 and 10 show results for some physical conditions across the cloud. The ionization structure of iron is shown in Figure 9, and the total and Fe II cooling are shown in Figure 10. The plotted quantities appear discontinuous at the hydrogen ionization front since conditions change rapidly there - these regions were actually well resolved in the calculations. Comparing the figures, it is clear that $\mathrm{Fe}$ II is by far the most important coolant behind the ionization front, where $\mathrm{Fe}$ II is the main fraction of iron. In fact, very near the hydrogen ionization front, Fe II carries essentially $100 \%$ of the total cooling and totally dominates conditions in the cloud. This occurs when cooling shifts away from the Balmer lines, which are becoming optically thick, and so the gas grows hotter and able to excited Fe II more effectively. The resulting spectrum of the cloud (continuum and emission lines, including all lines of $\mathrm{Fe}$ II) is shown in Figure 11.

Wills et al. (1985) found a simple three-level atom that reproduced the cooling of their model atom over some regimes of parameter space. We tried (unsuccessfully) to find a similar expression for the breadth of conditions we intend to model. The temperature and ionization profiles that result from the two treatments of the $\mathrm{Fe}$ II cooling were different enough that no meaningful comparisons between

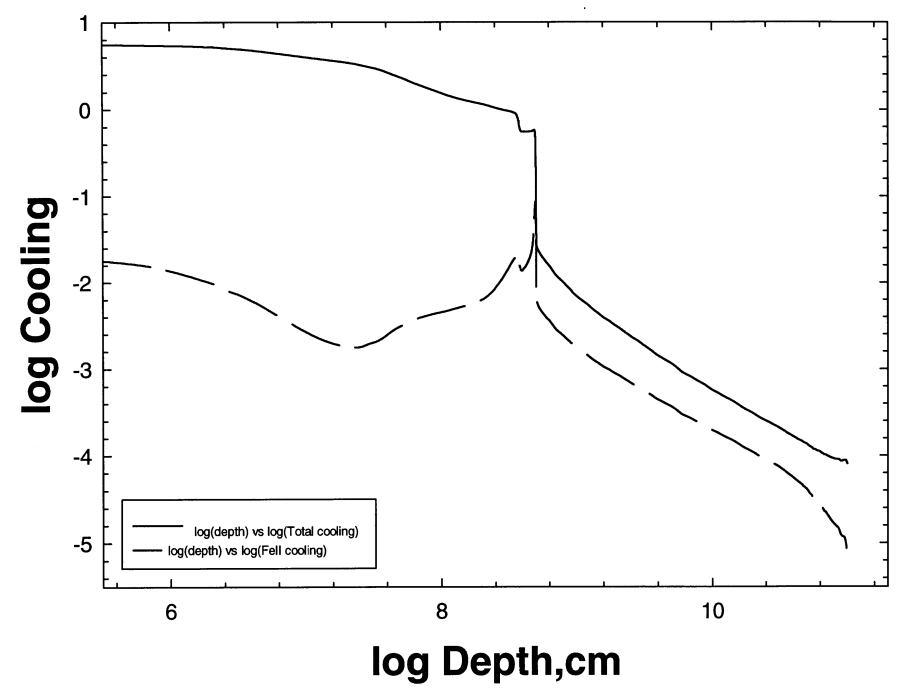

FIG. 10. - $\log$ total and Fe II cooling vs. depth within the cloud (cm) the Wills et al. cooling rates and our cooling could be made. We did make comparisons between predicted results for other lines and continua and found that they can be quite dramatic - for instance, the Balmer continuum emission is about $50 \%$ greater with the large atom due to larger temperatures in regions in which $\mathrm{Fe}$ II is a net heat source.

\subsection{Density and Flux Dependence}

Electron collisions are one of the main excitation mechanisms populating the $\mathrm{Fe}$ II atom. The resulting spectrum carries with it many clues to a cloud's electron density. For instance, some levels have no permitted decays to ground and build up relatively high populations. Forbidden or intercombination lines from such levels will become strong at relatively low densities. Transitions with metastable states as lower levels will become optically thick and thermalize at lower densities, too. Other lines come from levels that are depopulated by strong transitions and remain strong to high densities.

Here we investigate the density dependence of the spectrum. We first consider the UV and optical Fe II spectra produced at the high densities expected in quasar broad emission line regions. For the purposes of plotting, the lines are assumed to be broadened by a Gaussian macroturbulence with FWHM $=10^{3} \mathrm{~km} \mathrm{~s}^{-1}$, chosen to represent bulk cloud motion of separate clouds within the broad-line region. No microturbulence is assumed to exist within a cloud; the lines were only broadened by the Doppler width appropriate for the local electron temperature. Figure $12 a$ shows the UV and optical Fe II spectrum calculated at $n_{\mathrm{H}}=10^{9} \mathrm{~cm}^{-3}$, a flux of hydrogen-ionizing photons of $\log \left(\Phi_{\mathrm{H}}\right)=17.5 \mathrm{~cm}^{-2} \mathrm{~s}^{-1}$ (model 12a), and total hydrogen column density of $10^{24} \mathrm{~cm}^{-2}$. We used the baseline AGN continuum described in Korista et al. (1997), consisting of a UV bump peaking near $44 \mathrm{eV}$, a $f_{v} \propto v^{-1} \mathrm{X}$-ray power law, and the UV-to-optical spectral index $\alpha_{\mathrm{ox}}=-1.4$.

Figure $12 b$ presents the $\mathrm{Fe}$ II spectrum at a thousand times higher particle density, $n_{\mathrm{H}}=10^{12} \mathrm{~cm}^{-3}$, and similarly increased flux $\log \left(\Phi_{\mathrm{H}}\right)=20.5 \mathrm{~cm}^{-2} \mathrm{~s}^{-1}$ (model 12b). Both density and flux were increased by the same factor. This means that the ionization parameter is the same as in model 12a.

The spectra of models $12 \mathrm{a}$ and $12 \mathrm{~b}$ are quite different in the optical window, $3600 \AA<\lambda<5600 \AA$. A detailed study of the contributing transitions shows that the forbidden lines at these wavelengths are the strongest ones in model 12a, whereas the permitted optical lines are much stronger in the higher density model $12 \mathrm{~b}$. This agrees with Netzer (1988), who found that the forbidden lines become much weaker than the permitted optical lines at densities above $\log \left(n_{\mathrm{H}}\right)=9.5$. This provides an obvious density probe.

We find that typical blends of Fe II emission have critical densities, densities at which collisional rates equal radiative rates, varying between $\log \left(n_{\mathrm{H}}\right)$ of $9.5-10.5$, depending on other parameters of calculation (mainly the ionizing flux and microturbulent velocity, since these affect the line optical depth). Two broad blends of Fe II lines at 1000-1800 $\AA$ and a larger one at $2000-3600 \AA$ demonstrate this density dependence. The shortward feature becomes weaker relative to the longward one as density increases.

The ionization parameter also affects the spectrum. Model 12c (Fig. 12c) was computed with the same flux as in model 12a, $\log \left(\Phi_{\mathrm{H}}\right)=17.5 \mathrm{~cm}^{-2} \mathrm{~s}^{-1}$, and the same density as in model $12 \mathrm{~b}, n_{\mathrm{H}}=10^{12} \mathrm{~cm}^{-3}$. The ionization parameter 


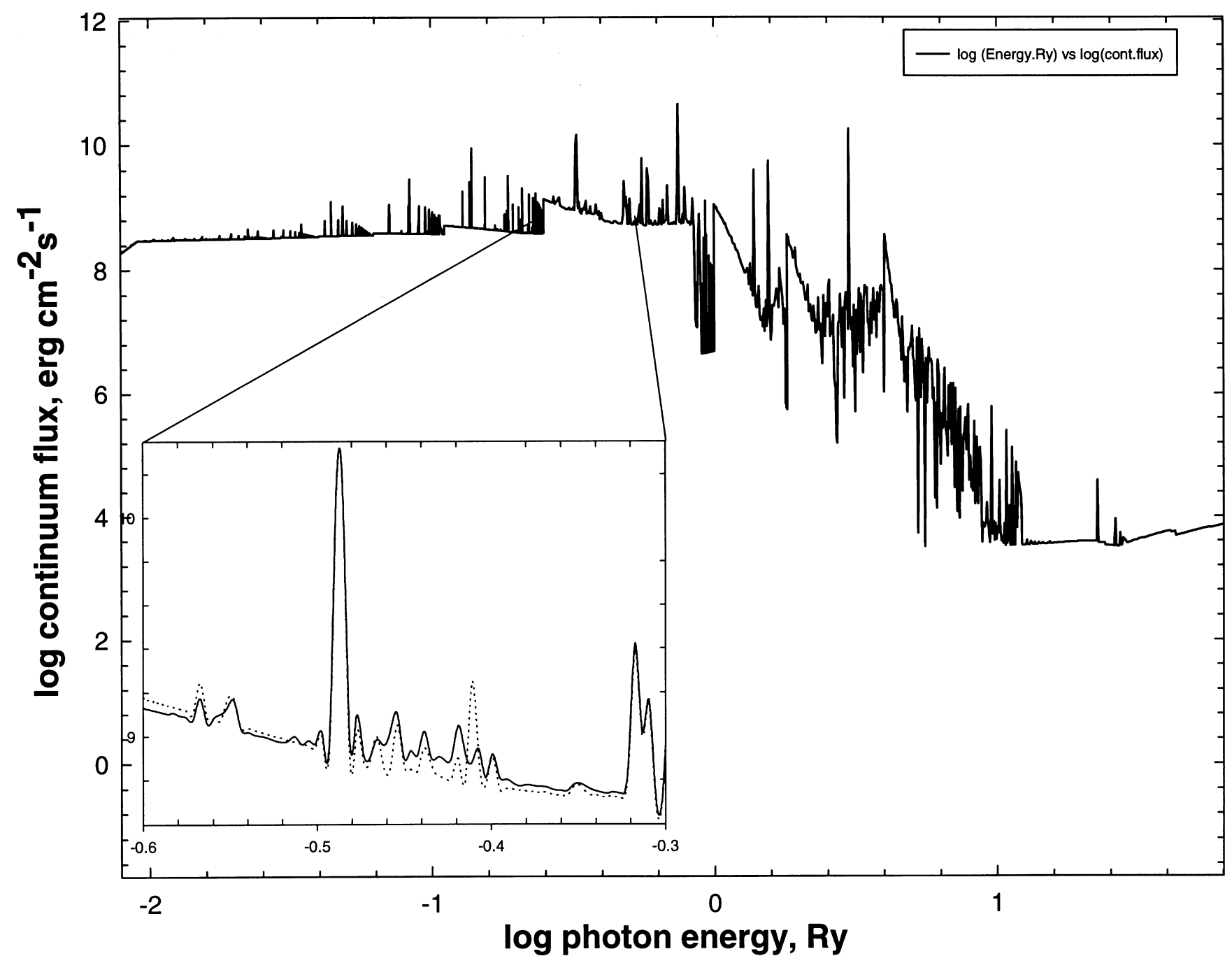

FIG. 11.- The diffuse emission from a cloud whose gas density is $\log n(\mathrm{H})=12 \mathrm{~cm}^{-3}$, ionization parameter is $\log U(\mathrm{H})=-3$, and column density is $\log N(\mathrm{H})=23 \mathrm{~cm}^{-2}$ and illuminated by an ionizing spectrum typical of quasars. The inset illustrates the comparison of the diffuse emission in the near-UV region with and without Fe II model included in the calculation. Note the significant bump in the spectral region corresponding to $2200-2700 \AA$ and the weaker Balmer continuum resulting from its destruction in the Fe II emitting zone. The dotted line is without pumping, and the solid line includes it.

in model 12c is 3 orders of magnitude lower than in models $12 \mathrm{a}$ and $12 \mathrm{~b}$, and the temperature of the gas cloud rapidly decreases with depth. The emission comes from only a relatively thin layer with lower optical depth, and therefore, the strongest resonance lines are relatively less saturated and remain efficient emitters. For instance, the strength of multiplets UV1 and UV2 is much larger than the emission that lies between the UV1 and UV2 (2400-2600 $⿱$ ) emission clusters. The weaker transitions in this wavelength interval are only strong relative to UV1 and UV2 when there is a significant contribution from the continuum pumping.

Two peaks around 2600 and $2400 \AA$ are the strongest features in all the models. They are mainly UV1 and UV2 multiplets, the first resonance multiplets of Fe II, although more than $3000 \mathrm{Fe}$ II lines have wavelengths between these two peaks. Hundreds of lines give significant contributions to the total Fe II emission, filling the gap between the two strongest peaks. Spectral features within this gap are produced by a pseudocontinuum from many lines of various multiplets. Depending on model parameters, tens or even hundreds of weak lines, both permitted and forbidden ones, may produce a higher total intensity at a given wavelength than the intensity of one strong permitted line. This makes it meaningless to associate any blend to a particular line in most cases and makes complete spectral synthesis mandatory.

\subsection{Dependence on Microturbulent Velocity}

Netzer \& Wills (1983) suggested that the microturbulent velocity could influence the intensity of $\mathrm{Fe}$ II emission. This is because broader line widths increase the efficiency of continuum pumping and decrease line optical depths. Effects of microturbulent velocity on emission spectra of various objects have been discussed recently by Alexander \& Netzer (1997) and Murray \& Chiang (1997). The main effect is to add an extra term to the line width, $v^{2}=k T / m+v_{t}^{2}$, and this has the effect of desaturating the line and allowing it to absorb radiation more efficiently.

We computed several BELR clouds with a density of $n_{\mathrm{H}}=10^{10} \mathrm{~cm}^{-3}$, a hydrogen-ionizing photon flux $\log \left(\Phi_{\mathrm{H}}\right)=18.5 \mathrm{~cm}^{-2} \mathrm{~s}^{-1}$, but various values of the microturbulent velocity. Model 13a (Fig. 13a) has thermal widths only, but the two others have added microturbulent velocities of $v_{t}=10 \mathrm{~km} \mathrm{~s}^{-1}$ (model 13b; Fig. 13b) and $v_{t}=100$ $\mathrm{km} \mathrm{s}^{-1}$ (model 13c; Fig. 13c). As the microturbulent velocity increases, the local line width does too, and pumping by 


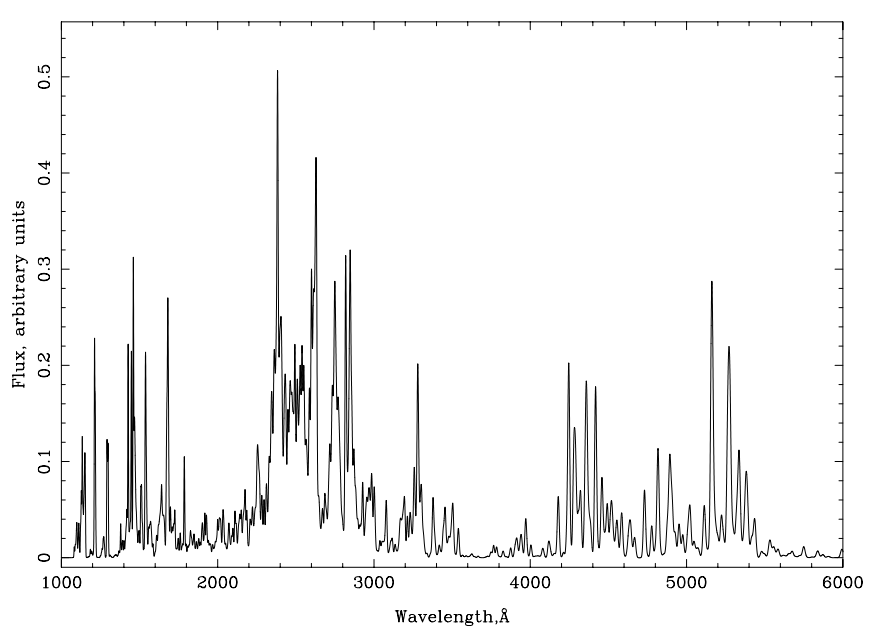

FIG. $12 a$

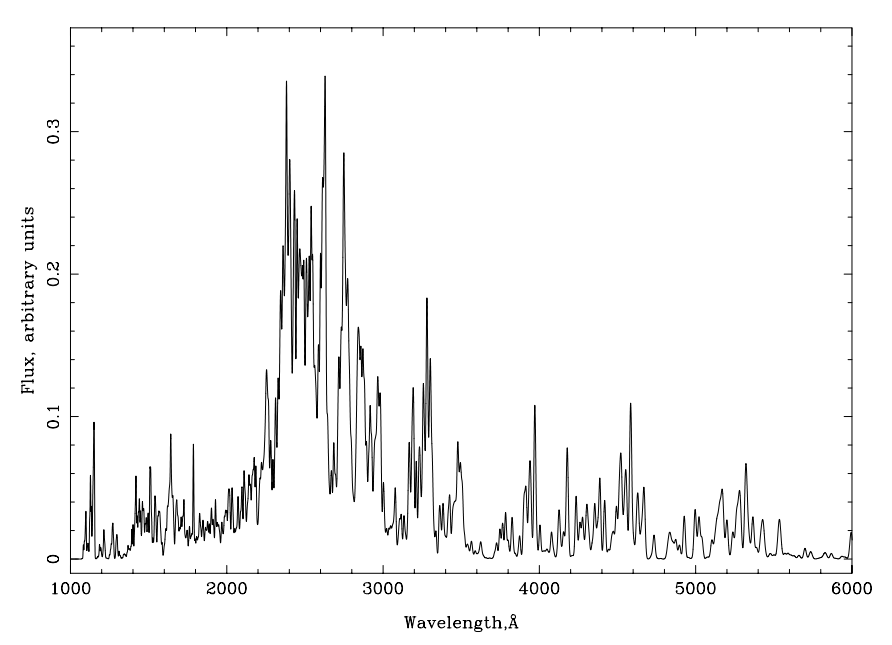

FIG. $12 b$

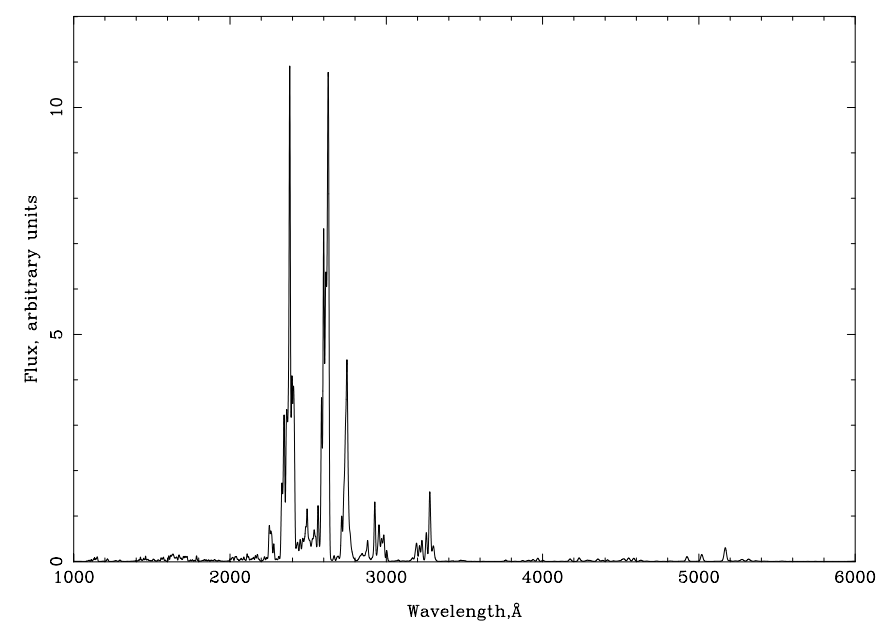

FIG. $12 c$

FIG. 12.-Theoretical UV and optical Fe II spectra calculated for the parameters expected in quasar broad emission line regions. The clouds are illuminated by a spectral energy distribution typical in AGNs, total hydrogen column density of $10^{24} \mathrm{~cm}^{-2}$, and solar abundances: (a) hydrogen density $n_{\mathrm{H}}=10^{9} \mathrm{~cm}^{-3}$, flux of hydrogen-ionizing photons $\log \left(\Phi_{\mathrm{H}}\right)=17.5 \mathrm{~cm}^{-2} \mathrm{~s}^{-1} ;(b) n_{\mathrm{H}}=10^{12} \mathrm{~cm}^{-3}, \log \left(\Phi_{\mathrm{H}}\right)=20.5 \mathrm{~cm}^{-2} \mathrm{~s}^{-1} ;(c) n_{\mathrm{H}}=10^{12} \mathrm{~cm}^{-3}, \log \left(\Phi_{\mathrm{H}}\right)=$ $17.5 \mathrm{~cm}^{-2} \mathrm{~s}^{-1}$.

both lines and the continuum becomes more effective. This selectively increases the intensities of lines arising from levels that can be pumped.

We found that relative intensities of UV and optical bumps of $\mathrm{Fe}$ II lines are also very sensitive to the microturbulent velocity $v_{t}$. Not only the form of the net Fe II emission is changed but also the full flux and profile of the $\mathrm{Fe}$ II emission. The main changes with increasing turbulent velocity are similar to those with increasing density. However, a high turbulent velocity enhances the UV spectrum of Fe II (1000-1800 and 2400-2600 ̊) far more than a high density does. This sensitivity to the assumed line profile is also a complication - the results are clearly sensitive to the exact treatment of the line broadening function and radiative transfer.

\subsection{The Fe Abundance}

Hamann \& Ferland (1993) suggested that highluminosity quasars have high abundances and proposed that $\mathrm{Fe}$ abundances could be used as a cosmological chronometer. To investigate the influence of $\mathrm{Fe}$ abundance, we did a series of calculations for a quasar BELR with a density $n_{\mathrm{H}}=10^{11} \mathrm{~cm}^{-3}$ and a hydrogen-ionizing photon flux of $\log \left(\Phi_{\mathrm{H}}\right)=18.5 \mathrm{~cm}^{-2} \mathrm{~s}^{-1}$. This corresponds to an ionization parameter of $10^{-3}$. Only the iron abundance was changed, by scale factors from $1 / 3$ (model $14 a$ ) to 5 times solar (model 14b).

Figure 14 shows spectra where the dashed line is for the smaller abundance and the solid line is for the higher one. A comparison between models $14 \mathrm{a}$ and $14 \mathrm{~b}$ shows that the relationship with $\mathrm{Fe} / \mathrm{H}$ is not a $1: 1$ relationship and that even different clusters of lines react differently to changes in the iron abundance. This is due to a competition between two effects as the abundance increases: the lines tend to become more saturated and so less efficient emitters, but the density of $\mathrm{Fe}$ II atoms increases, so they have greater effect. These dependencies show that a complete synthesis of the spectrum will be necessary to deduce reliable iron abundances and that the spectrum does not depend linearly on abundance.

\subsection{Ly $\alpha$ Pumping}

There has been a long and active debate concerning how important Ly $\alpha$ is for exciting $\mathrm{Fe}$ II (Brown et al. 1979; Penston et al. 1983; Johansson \& Jordan 1984; Netzer 1988). Netzer made calculations for a high-density 


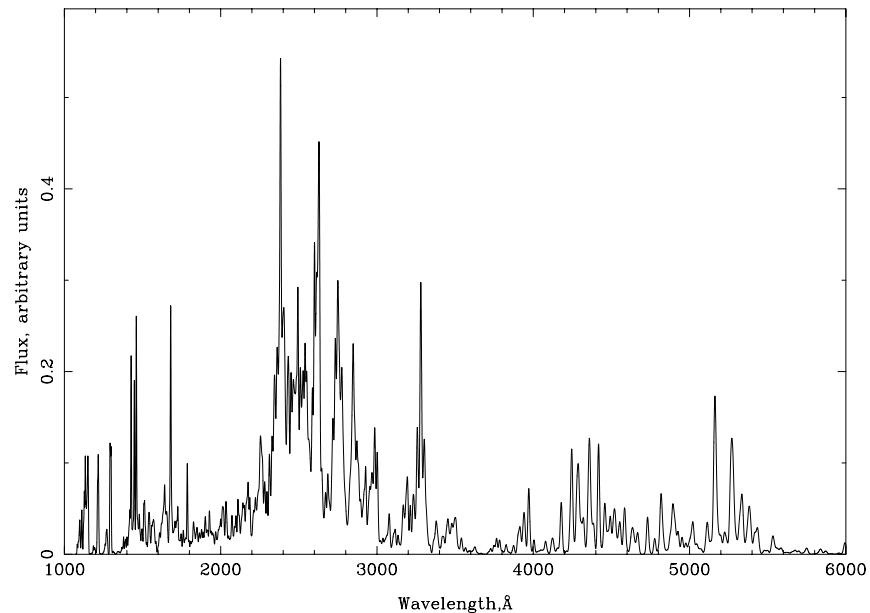

FIG. $13 a$

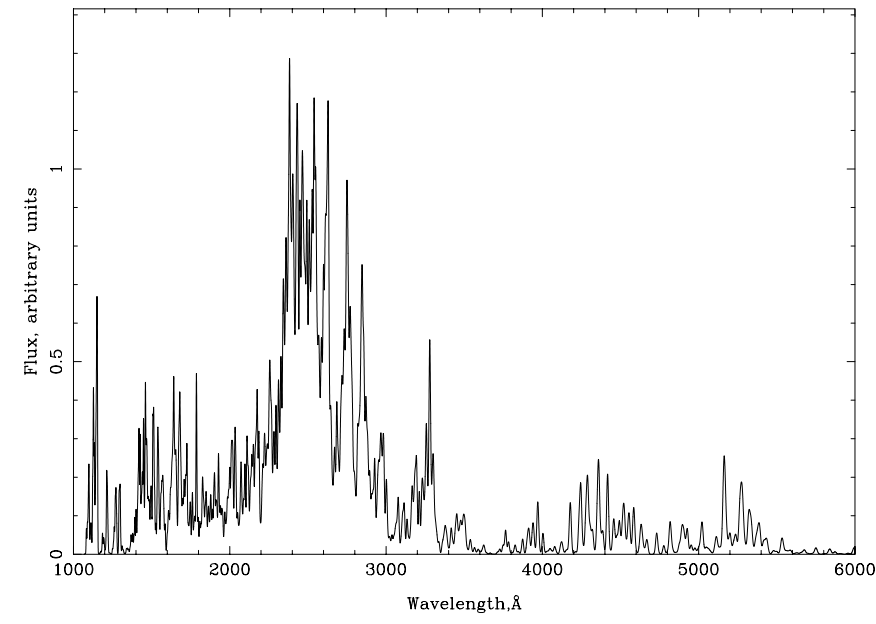

FIG. $13 b$

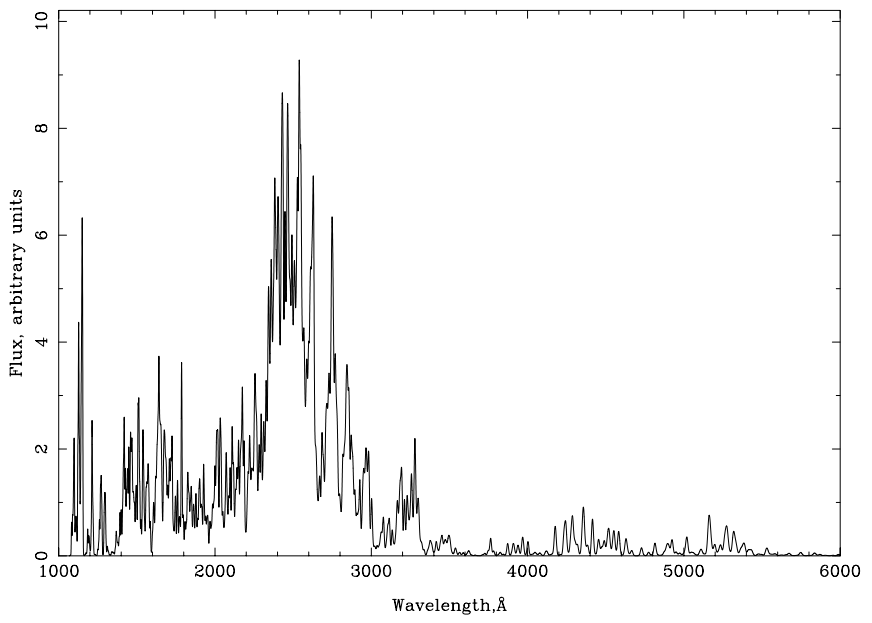

FIG. $13 c$

Fig. 13. - The same as Fig. 12 but for $n_{\mathrm{H}}=10^{10} \mathrm{~cm}^{-3}, \log \left(\Phi_{\mathrm{H}}\right)=18.5 \mathrm{~cm}^{-2} \mathrm{~s}^{-1}:(a)$ microturbulent velocity $v_{t}=0 \mathrm{~km} \mathrm{~s}$; $\left(\right.$ b) $v_{t}=10 \mathrm{~km} \mathrm{~s}$; (c) $v_{t}=100 \mathrm{~km} \mathrm{~s}^{-1}$

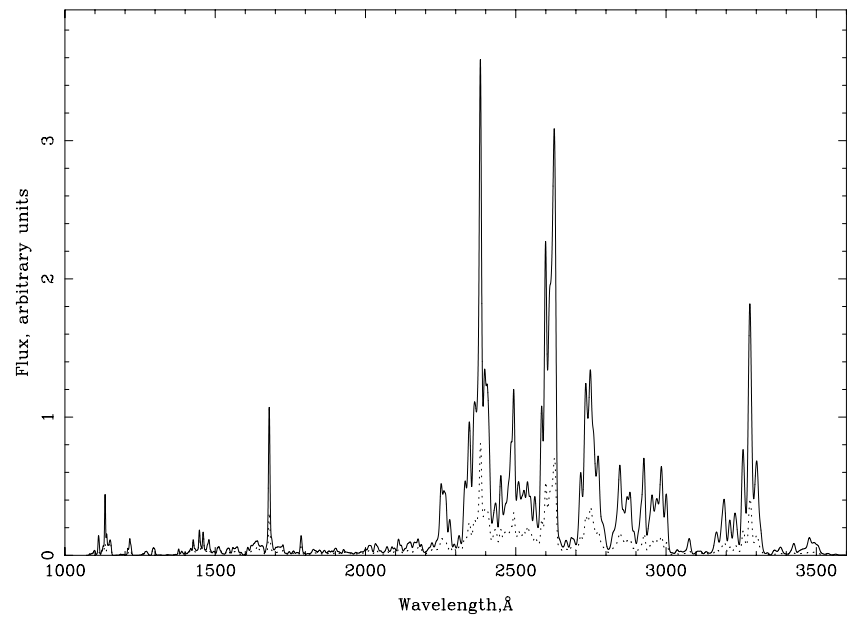

FIG. 14.-The same as Fig. 12 but for $n_{\mathrm{H}}=10^{11} \mathrm{~cm}^{-3}, \log \left(\Phi_{\mathrm{H}}\right)=18.5$ $\mathrm{cm}^{-2} \mathrm{~s}^{-1}$ : model $a, \mathrm{Fe}$ abundance $1 / 3$ solar; model $b, \mathrm{Fe}$ abundance 5 times solar.
$\tau(\operatorname{Ly} \alpha)=10^{8}$ gas where $\operatorname{Ly} \alpha$ fluorescence is included. These calculations showed that Ly $\alpha$ excites a few Fe II lines but the energy radiated in all of them is very small.

The importance of Ly $\alpha$ pumping is actually a question of the intrinsic width of $\operatorname{Ly} \alpha$, the excitation temperature or source function of $\mathrm{Ly} \alpha$, and the number of excited $\mathrm{Fe}^{+}$ levels energetically accessible. We find that this process can be very efficient for some conditions. The greater the number of levels that can contribute, the more effectively Ly $\alpha$ pumping works. The most important condition for efficient Ly $\alpha$ pumping is that the optical depth and resulting Ly $\alpha$ width be large enough to overlap with many optically thick Fe II lines. Otherwise, Fe II atoms cannot effectively absorb the Ly $\alpha$ photons.

The full non-LTE level populations of hydrogen are determined self-consistently for the local conditions, as described in Ferguson \& Ferland (1997) and the papers they cite. The width of $\operatorname{Ly} \alpha$ is calculated by CLOUDY using equation (3) above. The model hydrogen atom computes level populations for $2 s$ and $2 p$ independently, including the 


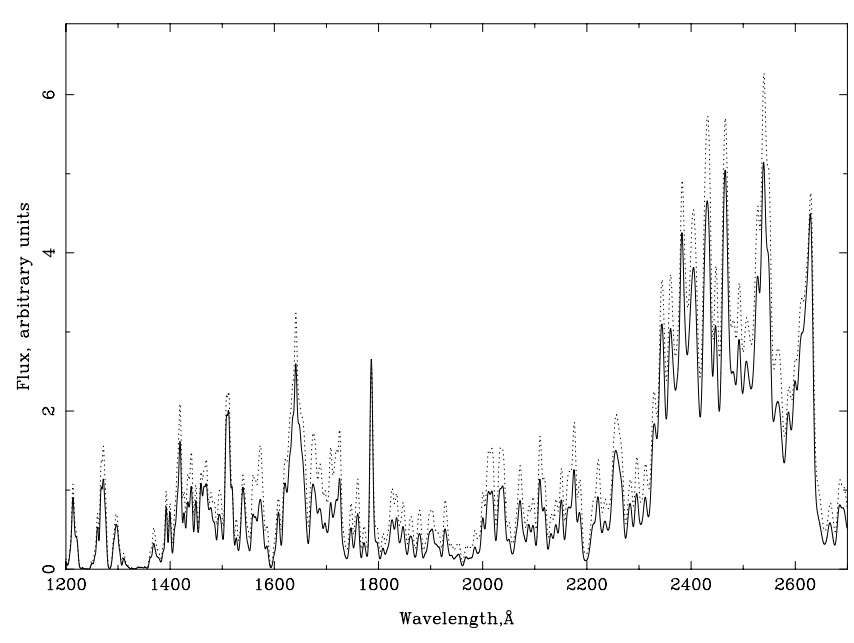

FIG. 15.-The solid line represents the Fe II spectrum predicted when the effects of $L y \alpha$ pumping are turned off. The dashed line shows the predicted spectrum with the inclusion of these effects. A microturbulent velocity of $100 \mathrm{~km} \mathrm{~s}^{-1}$ was assumed in both cases.

effects of $\mathrm{Ly} \alpha$ loss onto $\mathrm{Fe}^{+}$. We assume that the $\mathrm{Ly} \alpha$ source function is given by the Planck function at $1 s-2 p$ excitation temperature. The optical depth determines the line width as outlined by Elitzur \& Ferland (1986). Using theoretical calculations of the profiles of optically thick Ly $\alpha$ lines made by Adams (1972), we select all of the optically thick Fe II lines which have the wavelengths within the Ly $\alpha$ profile and determine the intensities of Ly $\alpha$ emission at the corresponding energies. These intensities are then used to calculate the coefficients of the induced transitions due to Ly $\alpha$ radiation. Ly $\alpha$ pumping is significant in populating the corresponding upper levels of the $\mathrm{Fe}$ II atom if these coefficients are significant compared to the collisional excitation and continuum pumping coefficients. The subsequent cascades of the spontaneous transitions change the populations of the lower levels and therefore the whole spectrum of the Fe II emission.

We computed a BELR with the Mathews \& Ferland (1987) continuum, solar abundances, a column density of $10^{23} \mathrm{~cm}^{-2}, U=10^{-2}$, a hydrogen density $n_{\mathrm{H}}=10^{12} \mathrm{~cm}^{-3}$, and microturbulent velocity of $100 \mathrm{~km} \mathrm{~s}^{-1}$ and did two calculations, with Ly $\alpha$ pumping switched on and off, to determine its effects. We found that the spectrum between 1000 and $2700 \AA$ (shown on Fig. 15) is most sensitive to Ly $\alpha$ pumping. It can be seen that Ly $\alpha$ pumping increases the full flux in the UV region, thus making many emission lines stronger. Ly $\alpha$ pumping does not affect some lines at all.

In general, $L y \alpha$ pumping is most effective for transitions whose lower level is $a^{4} D$ and upper level is $u^{4} D^{o}$. Increasing the microturbulent velocity allows more optically thick $\mathrm{Fe}$ II transitions to be pumped by the wider Ly $\alpha$. In particular, more upper states, including $u^{4} P^{o}, x^{6} F^{o}$, and $v^{5} F^{o}$ (which are energetically close to $u^{4} D^{o}$ ) can become acces- sible. Another consequence is that the gas tends to be hotter when Ly $\alpha$ pumping is included. This is the result of the process depositing electrons into very highly excited states that can then be collisionally de-excited.

\section{SUMMARY AND THE FUTURE}

Clearly the spectrum of the Fe II atom is rich with details, and we have only begun to harvest the information that is present. This paper lays the foundations for others that will explore detailed environments more fully. Here we have described the methods and assumptions that we have used to incorporate a large model $\mathrm{Fe}$ II atom into CLOUDY. The highlights include the following:

We have now incorporated a 371 level $\mathrm{Fe}^{+}$atom into CLOUDY. This is the largest atom that can realistically be verified and used in today's computer environments. For example, a typical BELR calculation takes several tens of minutes on a high-end processor without the $\mathrm{Fe}$ II atom but tens of hours with it. The atom is designed so that we can readily expand it as computers become faster.

All transitions in the atom are now transferred using escape probabilities. Destruction of lines by background opacities and effects of overlapping lines and pumping are all included as excitation and de-excitation processes. These are incorporated in such a manner that the eventual incorporation of exact radiative transfer techniques will be possible when computers are fast enough.

Transition probabilities for all lines are included using the best theoretical and experimental data. Temperature dependent collision strengths were fitted to published data where possible. For others we used various $\bar{g}$ approximations. New sources of both types of data will become available in coming years, and we have incorporated the current data set in a way that can be easily changed.

The full equations of statistical equilibrium are solved as a function of depth, and the results of the model atom are fed back into the rest of CLOUDY. The solution is fully self-consistent, conserves energy, and includes the effects of $\mathrm{Fe}$ II emission on the temperature and ionization of the cloud. These effects are profound for some conditions.

We presented several example calculations that illustrate how rich a diagnostic the Fe II spectrum is for conditions in line-emitting gas. Future papers will explore this in more detail for specific environments.

Research in nebular astrophysics at the University of Kentucky is supported by the National Science Foundation by grant AST 96-17083, by NASA through NRA-96-04GSFC-123, and by STScI through AR 07526. The work of F. H. was supported by NASA grant NAG 5-3234. We are grateful to the Center for Computational Sciences at the University of Kentucky and the Exemplar supercomputer support team. We thank Moshe Elitzur for helpful discussions and the anonymous referee for a thoughtful report.
Adams, T. F. 1972, ApJ, 174, 439

Alexander, T., \& Netzer, H. 1997, MNRAS, 284, 967

Brown, A., Jordan, C., \& Wilson, R. 1979, in The First Year of IUE, ed.

A. Wills (London: Univ. College, London), 232

Elitzur, M. 1984, ApJ, 280, 653

. 1992, Astronomical Masers (Dordrecht: Kluwer)

Elitzur, M., \& Ferland, G. J. 1986, ApJ, 305, 35

Elitzur, M., \& Netzer, H. 1985, ApJ, 291, 464

\section{REFERENCES}

Ferguson, J. W., \& Ferland, G. J. 1997, ApJ, 479, 363

Ferland, G. J. 1996, HAZY, a Brief Introduction to CLOUDY, University of Kentucky Physics Department Internal Report

Ferland, G. J., Korista, K. T., Verner, D. A., Ferguson, J. W., Kingdon, J. B., \& Verner, E. M. 1998, PASP, in press

Ferland, G. J., \& Persson, S. E. 1989, ApJ, 347, 656

Ferland, G. J., Peterson, B. M., Horne, K., Welsh, W. F., \& Nahar, S. N. 1992, ApJ, 387, 95 
Fuhr, J. R., Martin, G. A., \& Wiese, W. L. 1988, J. Phys. Chem. Ref. Data, 17, Suppl. 4

Giridhar, S., \& Arellano Ferro, A. 1995, Rev. Mexicana Astron. Astrofis., 31,23

Hamann, F., \& Ferland, G. 1993, ApJ, 418, 11

Hauschildt, P. H., Baron, E., Starrfield, S., \& Allard, F. 1996, ApJ, 462, 386

Hoeflich, P., Wheeler, J. C., \& Thielemann, F. K. 1998, ApJ, 495, 617

Johansson, S. 1978, Phys. Scripta, 18, 217

Johansson, S., \& Jordan, C. 1984, MNRAS, 210, 239

Kalkofen, W. 1984, Methods in Radiative Transfer (Cambridge: Cambridge Univ. Press)

Korista, K., Baldwin, J., Ferland, G., \& Verner, D. 1997, ApJS, 108, 401

Kozma, C., \& Fransson, C. 1998a, ApJ, 496, 946 1998 b, ApJ, 497, 431

Kurucz, R. L. 1995, Kurucz CD-ROM 23 (Cambridge: Smithsonian Astrophysical Observatory)

Lanz, T., Hubeny, I., \& Heap, S. R. 1997, ApJ, 485, 843

Li, H., \& McCray, R. 1996, ApJ, 456, 370

Mathews, W. G., \& Ferland, G. J. 1987, ApJ, 323, 456

Mewe, R. 1972, A\&AS, 20, 215
Mihalas, D. 1978, Stellar Atmospheres (2d ed.; W. H. Freeman: San Francisco)

Murray, N., \& Chiang, J. 1997, ApJ, 474, 91

Nahar, S. N. 1995, A\&A, 293, 967

Netzer, H. 1988, in Physics of Formation of Fe II Lines Outside LTE, ed. R. Viotti, A. Vittone, \& M. Friedjung (Dordrecht: Reidel), 247

. 1990, in Active Galactic Nuclei, ed. T. J.-L. Courvorsier \& M. Mayor (Saas-Fee Advanced Course 20; Springer: Berlin), 57 Netzer, H., \& Wills, B. J. 1983, ApJ, 275, 445

Osterbrock, D. E. 1989, Astrophysics of Gaseous Nebulae and Active Galactic Nuclei (Millalley: University Science Books)

Penston, M. V. 1987, MNRAS, 229, 1P

Penston, M. V., et al. 1983, MNRAS, 202, 833

Phillips, M. M. 1978a, ApJS, 38, 187 1978b, ApJ, 226, 736

Quinet, P., Le Dourneuf, M., \& Zeippen, C. J. 1996, A\&AS, 120, 361

Wills, B. J., Netzer, H., \& Wills, D. 1985, ApJ, 288, 94

Young, P. R., Landi, E., \& Thomas, R. J. 1998, A\&A, 329, 291

Zhang, H. L., \& Pradhan, A. K. 1995, A\&A, 293, 953 